Rev. Inst. Flor. v. 29 n. 2 p. 199-209 dez. 2017

http://dx.doi.org/10.24278/2178-5031.201729205

ISSN impresso 0103-2674/on-line 2178-5031

\title{
RENDIMENTO DO PROCESSAMENTO DE TRÊS ESPÉCIES FLORESTAIS AMAZÔNICAS ${ }^{1}$
}

\section{PROCESSING YIELD OF THREE AMAZON FOREST SPECIES}

\author{
Nayanna de Nazaré Brito FREITAS ${ }^{2,4}$; Iêdo Souza SANTOS ${ }^{3}$; \\ Paulo Cezar Gomes PEREIRA ${ }^{3}$
}

\begin{abstract}
RESUMO - A crescente procura da matéria-prima vem ocasionando o encarecimento do produto, desse modo, as empresas devem possuir um processo de desdobramento que obtenha melhor aproveitamento, levando ao aumento do rendimento na produção. $\mathrm{O}$ objetivo deste trabalho foi avaliar o coeficiente de rendimento volumétrico de três espécies florestais amazônicas: Hymenolobium petraeum, Goupia glabra e Manilkara spp. Foram selecionadas cinco toras de madeira aleatoriamente para cada espécie analisada, o volume da madeira em tora foi obtido pelos métodos de Smalian e Francon. Posteriormente, foi obtido o volume da madeira processada, medindo-se as dimensões de cada peça serrada correspondente a cada uma das toras. O cálculo de rendimento foi determinado pela razão entre o volume de madeira serrada e o volume da madeira em tora. A análise estatística foi realizada através do teste de Tukey com o intuito de avaliar as médias levando em consideração um delineamento inteiramente casualizado, utilizando o programa ASSISTAT Versão 7.7. O rendimento médio das três espécies estudadas foi equivalente a $50,83 \%$, valor satisfatório para as condições da região, a qual não apresenta nível de automação elevado e nem mão de obra qualificada.
\end{abstract}

Palavras-chave: rendimento volumétrico; madeira nativa; tecnologia da madeira.

\begin{abstract}
The growing demand for raw materials is causing the increasing of the product, thus, companies must have a process of unfolding that obtains better exploitation, succeeding the increase in yield in production. The aim of this work was to evaluate the coefficient of volumetric yield of Amazon forests species: Hymenolobium petraeum, Goupia glabra and Manilkara spp. Five wood logs were randomly selected for each species analyzed, the observed volume of wood was based on the geometric cubing methods (Smalian and Francon). Subsequently the measurement of the volume of processed wood was carried out by the romaneio procedure, the calculation of the yield of the volume of each log was determined before the unfolding process. The statistical analysis was conducted through Tukey test aimed to evaluate the averages taking into consideration an entirely randomized design, using the program version 7.7. The average yield of the three studied species has been equivalent to $50.83 \%$ satisfactory value for the conditions of the region, which does not present high levels of automation and not skilled labor.
\end{abstract}

Keywords: volumetric yield; native wood; wood technology.

\footnotetext{
'Recebido para análise em 15.04.2017. Aceito para publicação em 01.12.2017.

2Discente do Curso de Engenharia Florestal da Universidade do Estado do Pará, Paragominas, PA, Brasil.

${ }^{3}$ Universidade do Estado do Pará, Departamento de Tecnologia da Madeira, Rua PA-125, s/n, Bairro Angelim, 68625-000, Paragominas, PA, Brasil.

${ }^{4}$ Autor para correspondência: Nayanna de Nazaré Brito Freitas - nayyyfreitas@hotmail.com
} 


\section{INTRODUÇÃO}

O Brasil possui um importante papel para o setor florestal mundial, o mesmo aborda setores que utilizam a madeira como insumo principal, como indústrias de papel e celulose, laminação e madeira serrada para diversos fins (Juvenal e Mattos, 2002).

A região amazônica possui um alto potencial madeireiro, sendo considerada uma das principais produtoras de madeiras tropicais do mundo, o segmento florestal impulsiona de forma direta a economia de dezenas de municípios da Amazônia. No estado do Pará, que possui áreas extensas de florestas ricas em espécies arbóreas com potencial de comercialização, o setor madeireiro foi responsável por produzir aproximadamente 2 milhões de metros cúbicos de madeira nativa em tora, comercializando mais de 1,3 milhões de metros cúbicos de outros produtos e subprodutos florestais no ano de 2015 (Instituto do Homem e Meio Ambiente da Amazônia IMAZON, 2010; Pará, 2016).

Quanto à classificação das empresas, a maioria das indústrias responsáveis por toda essa produção pode ser classificada como de pequeno e médio porte. Batista et al. (2015) atentam em seu trabalho que a grande maioria das empresas nacionais de pequeno e médio porte não apresentam informações precisas do desempenho operacional da sua produção. Os autores esclareceram que a falta de técnica é um dos motivos que impede o desenvolvimento dos empreendimentos, afetando, consequentemente, a eficiência e rendimento da produção.

Desse modo, o rendimento em madeira serrada mostra ser a principal variável para analisar o sucesso do empreendimento, sendo este influenciado por diversos fatores como: características das espécies, o layout da empresa, maquinário utilizado, o nível de automação, o sistema de desdobro, mão de obra e, principalmente, o diâmetro das toras, os modelos de corte e as dimensões das peças serradas (Garcia , 2013; Vital, 2013; Batista et al., 2015).

Sabe-se que há uma necessidade de aperfeiçoar o processo de desdobramento, a fim de promover um maior aproveitamento das toras com menor geração de resíduos, principalmente na região amazônica, onde as técnicas de exploração das florestas naturais são ineficientes devido à falta de planejamento e de técnicas apropriadas (Murara Junior et al., 2013).

Para uma madeireira se manter competitiva no mercado, deve apresentar um bom planejamento operacional e uma linha de produção eficiente e eficaz, desta forma, a empresa que possui conhecimento dos seus recursos, e sabe utilizá-los, detém como resultado um desempenho progressivo levando ao rendimento (Manhiça, 2010).

As espécies analisadas foram 0 angelim-pedra (Hymenolobium petraeum Ducke), cupiúba (Goupia glabra Aubl.) e maçaranduba (Manilkara spp.). Elas desempenham papel de vital importância na indústria madeireira, uma vez que possuem inúmeras aplicações, sem dificuldades para serrar, aplainar, tornear e realizar acabamentos, além de apresentarem alta densidade, boa durabilidade e resistência mecânica (Hiramatsu, 2008).

O presente estudo baseia-se na Resolução Conselho Nacional do Meio Ambiente - CONAMA No 474 de 06 de abril de 2016 (Brasil, 2016), a mesma dispõe sobre o coeficiente de rendimento volumétrico de produtos e subprodutos florestais madeireiros de origem nativa, este designado ao aproveitamento para transformação das matérias-primas de tora em madeira serrada. Nesse contexto, o trabalho teve como objetivo avaliar o rendimento de três espécies amazônicas: Hymenolobium petraeum Ducke, Goupia glabra Aubl. e Manilkara spp., bem como determinar o Coeficiente de Rendimento Volumétrico médio.

\section{MATERIAL E MÉTODOS}

O estudo foi realizado em uma madeireira do município de São Miguel do Guamá, aproximadamente $150 \mathrm{~km}$ de Belém, capital do estado do Pará, com as seguintes coordenadas geográficas W: 47²9'30" S: 01'36'35'. Durante o estudo, foram utilizadas cinco toras de cada espécie, totalizando quinze toras, em que se realizaram os cálculos de volumes (Equações 1, 2 e 3) e rendimento (Equação 4). 
FREITAS, N. de N.B.; SANTOS, I.B.; PEREIRA, P.C.G. Rendimento de madeiras nativas.

Foi realizada a análise estatística para verificar as médias dos rendimentos volumétricos levando em consideração um Delineamento Inteiramente Casualizado - DIC, agrupando as toras por quantidade de espécies (tratamento), com o número de cinco repetições por tratamento (cinco toras por espécie).

\subsection{Cubagem das Toras}

A tora apresentou formato geométrico semelhante a um cilindro, no pátio foi obtido o diâmetro e comprimento, imediatamente foi realizado o método de cubagem geométrica utilizando a fórmula de Smalian de acordo com a Resolução CONAMA № 411 de 06 de maio de 2009 (Brasil, 2009), como apresentado na Equação 1.

$$
V=\left[\left(\frac{D b}{100}\right)^{2}+\left(\frac{D t}{100}\right)^{2}\right] \frac{\pi}{8} L m
$$

Em que:

$\mathrm{V}=$ Volume da tora $\left(\mathrm{m}^{3}\right)$;

$\mathrm{Db}=$ Diâmetro médio da base $(\mathrm{cm})$;

$\mathrm{Dt}=$ Diâmetro médio do topo $(\mathrm{cm})$;

$\mathrm{Lm}=$ Comprimento médio $(\mathrm{m})$.

A cubagem Francon é utilizada quando ocorre perda de volume pelo processo de pré-produção antes da realização do desdobro principal, para obter o volume considera-se que a tora seja um cilindro no qual se utiliza a seção da extremidade que possui a menor área, conforme demonstrado na Equação 2.

$$
V=\left[\frac{(C)^{2}}{4}\right] L
$$

Em que:

$\mathrm{V}=$ Volume da tora $\left(\mathrm{m}^{3}\right)$;

$\mathrm{C}=$ Circunferência $(\mathrm{m})$;

$\mathrm{L}=$ Comprimento (m).

\subsection{Romaneio}

Mensurou-se a largura e espessura das extremidades e centro de cada peça com auxílio de uma trena métrica, a medida de comprimento foi obtida com a ajuda de uma fita métrica, posteriormente os dados foram usados para obtenção do volume de madeira serrada (Equação 3).

$$
V m s=[(M L)(M E)(L)]
$$

Em que:

Vms = Volume de madeira serrada (m);

$\mathrm{ML}=$ Média das larguras (m);

$\mathrm{ME}=$ Média das espessuras (m);

$\mathrm{L}=$ Comprimento $(\mathrm{m})$.

\subsection{Rendimento}

Para a análise do rendimento foram utilizadas as cinco toras, foi determinado o volume de cada uma de acordo com a equação de cubagem geométrica. Após as medições, as toras foram encaminhadas para o processo de desdobro, posteriormente ao processo de desdobro, as peças originadas das toras foram medidas e calculou-se o volume da madeira serrada de cada tora. Os valores obtidos foram organizados no software Microsoft Office Excel 2013 e, em seguida, foi realizado o cálculo de rendimento de acordo com a Equação 4.

$$
R m s=\left[\left(\frac{V m s}{V t}\right)\right] 100
$$

Em que:

Rms = Rendimento em madeira serrada (\%);

Vms = Volume de madeira serrada $\left(\mathrm{m}^{3}\right)$;

$\mathrm{Vt}=$ Volume com cascada tora $\left(\mathrm{m}^{3}\right)$.

\subsection{Análise Estatística}

Os cálculos foram efetuados com o auxílio do software Microsoft Office Excel, e as análises estatísticas foram efetuadas através do programa ASSISTAT Versão 7.7.

\section{RESULTADOS E DISCUSSÃO}

Nas Figuras 1, 2 e 3, encontram-se os rendimentos das três espécies analisadas; na Tabela 1, são apresentados os diâmetros das toras e os seus respectivos comprimentos, e as Tabelas 2 e 3 referem-se ao rendimento volumétrico. A maioria das toras apresentou rendimento acima do estabelecido pela Resolução CONAMA 474/2016 (Brasil, 2016), que estabelece um coeficiente de rendimento volumétrico - CRV de 35\%. Esses altos rendimentos podem ser resultado de fatores como erros de medições no campo. Tonini e Ferreira (2004) apresentam outras influências como o tipo de serra, reaproveitamento de aparas e costaneiras e da metodologia usada na avaliação do rendimento. 
FREITAS, N. de N.B.; SANTOS, I.B.; PEREIRA, P.C.G. Rendimento de madeiras nativas.

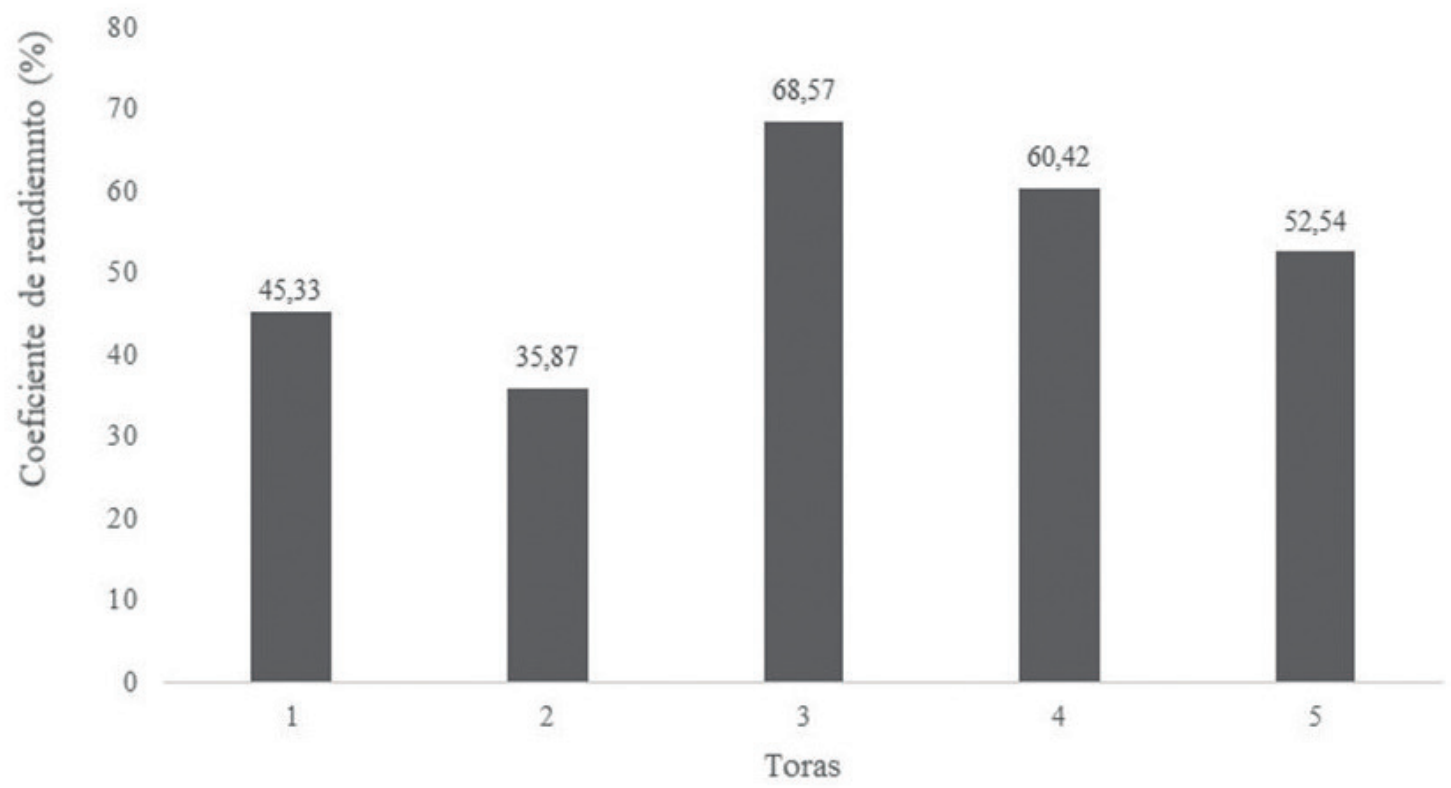

Figura 1. CRV de angelim-pedra por tora.

Figure 1. Coefficient of volumetric yield of angelim-pedra per log.

Tabela 1. Diâmetro e comprimento das toras utilizados no cálculo de rendimento.

Table 1. Diameter and length of the logs used to calculate the yield.

\begin{tabular}{ccccc}
\hline & TORAS & ${ }^{1}$ DB $(\mathrm{m})$ & ${ }^{2} \mathrm{DT}(\mathrm{m})$ & ${ }^{3} \mathrm{~L}(\mathrm{~m})$ \\
\cline { 2 - 4 } ANGELIM-PEDRA & 1 & 0,81 & 0,73 & 4,29 \\
& 2 & 0,81 & 0,78 & 5,82 \\
& 3 & 0,56 & 0,54 & 5,46 \\
CUPIÚBA & 4 & 0,85 & 0,87 & 6,31 \\
& 5 & 0,81 & 0,67 & 5,95 \\
\hline & 1 & 0,49 & 0,48 & 4,56 \\
MAÇARANDUBA & 2 & 0,51 & 0,50 & 4,13 \\
& 3 & 0,56 & 0,52 & 7,76 \\
& 4 & 0,61 & 0,56 & 6,20 \\
& 5 & 0,51 & 0,47 & 5,19 \\
\hline & 1 & 0,57 & 0,52 & 6,74 \\
& 2 & 0,58 & 0,50 & 5,37 \\
& 3 & 0,51 & 0,47 & 6,62 \\
& 4 & 0,63 & 0,53 & 7,41 \\
& 5 & 0,56 & 0,50 & 6,91
\end{tabular}

${ }^{1}$ Diâmetro base; ${ }^{2}$ Diâmetro topo; ${ }^{3}$ Comprimento.

${ }^{1}$ Base diameter; ${ }^{2}$ Diameter top; ${ }^{3}$ Length. 
FREITAS, N. de N.B.; SANTOS, I.B.; PEREIRA, P.C.G. Rendimento de madeiras nativas.

Coeficiente de Rendimento Volumétrico CRV foi determinado conforme recomenda a resolução CONAMA no 474/2016. Na Tabela 2, observa-se que as médias de rendimento estão acima do valor quando comparadas com o estipulado pela resolução, isto pode ser explicado pelo fato de terem sido considerados nos cálculos de madeira serrada os produtos e subprodutos (aproveitamento) gerados a partir da tora.

Observa-se que as espécies angelim-pedra e maçaranduba apresentaram coeficientes de variação altos, $24,24 \%$ e $24,67 \%$, respectivamente, a espécie cupiúba apresentou coeficiente de variação de 10,99\% (Smalian) e 9,03\% (Francon) (Tabela 2), considerada uma variação baixa, mas com alta precisão, segundo Pimentel Gomes (2000). Analisando os coeficientes de variação entre os métodos de cubagem, nota-se que o método de Smalian apresenta maior variação, isto ocorre devido ao maior volume ao analisar a tora como um todo, já que retrata o volume real da tora, enquanto o método Francon obtém seu volume a partir de uma peça retangular, pois elimina as costaneiras deixando a tora o mais homogênea possível, assim o rendimento aparenta ser inversamente proporcional ao volume da tora.

A espécie angelim-pedra apresentou rendimento entre 35,87 a $68,57 \%$ (média 52,55\% $\pm 12,74)$ para o rendimento em que o volume da tora foi obtido pelo método Smalian, e o rendimento pelo método de cubagem Franco, no valor de rendimento entre 45,33 a 68,57\% (média $67,08 \pm 16,19)$ (Tabela 2; Figura 1). No trabalho de Tonini e Ferreira (2004), o angelim-pedra foi a espécie que apresentou maior rendimento médio (66\%), os autores explicam que o valor encontrado é considerado alto para a região amazônica, situando-se acima dos valores determinados pela legislação, que na época era de 54,28\%, ressalta-se que os autores se basearam na Instrução Normativa IBAMA nº 01/1996.

Tabela 2. Rendimento volumétrico das espécies.

Table 2. Volumetric yield of the species.

\begin{tabular}{cccc}
\hline & \multicolumn{2}{c}{ RENDIMENTO - SMALIAN } & \\
\hline ESPÉCIES & $\mathrm{CRV}^{1}(\%)$ & $\mathrm{DP}^{2}$ & $\mathrm{CV}^{3}(\%)$ \\
\hline ANGELIM-PEDRA & 52,55 & 12,74 & 24,24 \\
CUPIÚBA & 57,35 & 6,30 & 10,99 \\
MAÇARANDUBA & 42,59 & 10,51 & 24,67 \\
\hline MÉDIA & 50,83 & \\
\hline ESPÉCIES & RENDIMENTO - FRANCON & $\mathrm{CV}(\%)$ \\
\hline ANGELIM-PEDRA & CRV $(\%)$ & DP & 24,14 \\
CUPIÚBA & 67,08 & 16,19 & 9,03 \\
MAÇARANDUBA & 62,43 & 5,64 & 21,47 \\
\hline MÉDIA & 48,56 & 10,42 & \\
\hline
\end{tabular}

${ }^{1}$ Coeficiente de Rendimento Volumétrico; ${ }^{2}$ Desvio-Padrão; ${ }^{3}$ Coeficiente de Variação.

${ }^{1}$ Coefficient of Volumetric Yield; ${ }^{2}$ Standard Deviation; ${ }^{3}$ Coefficient of Variation.

A espécie maçaranduba apresentou média de rendimento de $42,59 \% \pm 10,51$ por meio do método Smalian, e pelo Francon, rendimento médio de 48,56 $\pm 10,42$ (Tabela 2). A espécie demonstrou rendimento superior quando comparado aos dados encontrados na literatura, que variam entre 20 a $40 \%$, porém o rendimento médio para o desdobro das toras pode estar na faixa de 45 a 55\%, como sugerido por Danielle (2013). Neste estudo, somente as toras 1, 4 e 5 permaneceram dentro do indicado (Figura 2). Santos et al. (2017), ao analisarem essa mesma espécie, apresentaram valores médios de $46,04 \%$ de CRV. 


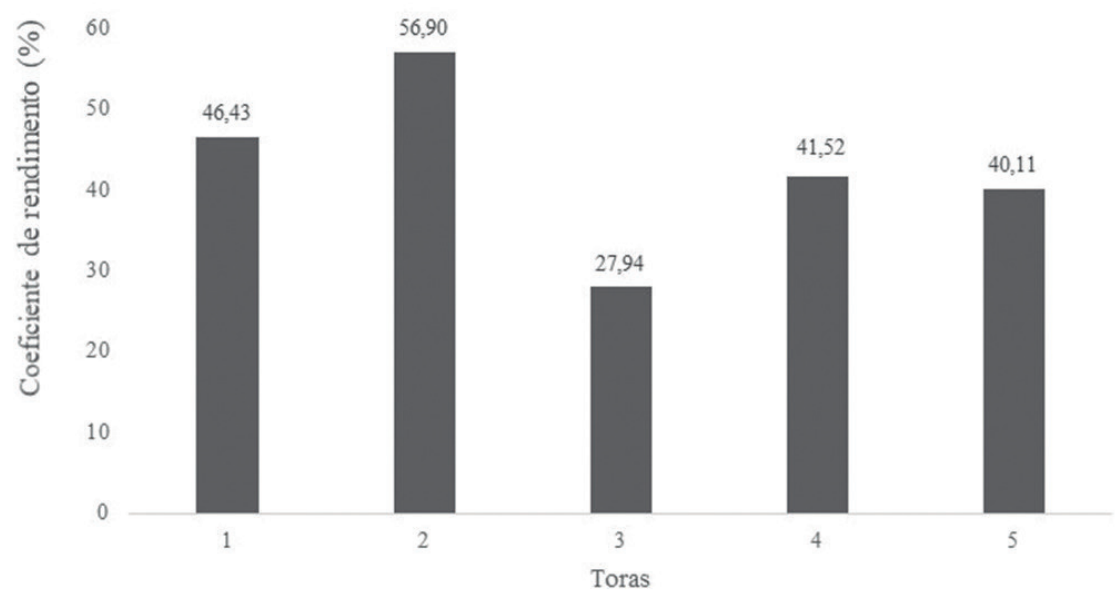

Figura 2. CRV de maçaranduba por tora.

Figure 2. Coefficient of volumetric yield of maçaranduba per log.

Iwakiri (1990) e Danielli (2013) analisaram a mesma espécie e apresentaram rendimento de $54,8 \%$ e 30,01\%, respectivamente. Melo et al. (2012), ao tratarem os altos rendimentos, explicam que o fato pode estar relacionado com o uso de maquinário no desdobramento da madeira; neste caso, afirma-se que o rendimento da produção está relacionado com a qualidade dos maquinários, bem como com a qualificação de mão de obra dos profissionais que atuam no processo da empresa.

A espécie cupiúba apontou maior média de rendimento, equivalente a $57,35 \% \pm 6,30$ no método geométrico e $62,43 \% \pm 5,64$ para Francon (Tabela 2; Figura 3), devido às toras apresentarem maiores dimensões e volume. Murara Junior et al. (2005) explicam que a qualidade da matéria-prima pode influenciar em até $70 \%$ no êxito da obtenção de um ótimo rendimento; desse modo, os autores afirmaram que o rendimento de madeira serrada aumenta de acordo com o incremento no diâmetro das toras utilizadas. Tonini e Ferreira (2004), estudando o rendimento do desdobro de toras das espécies cupiúba, caferana e angelim-pedra, para cupiúba apresentaram a média de rendimento igual a $52 \%$. No entanto, para essa mesma espécie, Santos et al. (2017) apresentaram valores médios de $47,77 \%$ de rendimento, esta variabilidade de resultado é reflexo da heterogeneidade peculiar das essências florestais amazônicas. Contudo, todos os valores apresentados são superiores ao $35 \%$ de CRV recomendado pela Resolução 474/2016 do CONAMA (Brasil, 2016).

O rendimento médio da empresa, analisado a partir das três espécies comercias, foi equivalente de 50,83\%, como observado na Figura 4. Quanto à tendência do rendimento, a espécie angelim-pedra foi aquela que se aproximou da média geral dos rendimentos analisados. Observou-se que a espécie cupiúba possui a tendência de aumento de rendimento, visto que suas toras foram as que apresentaram maiores diâmetros; entretanto, a espécie maçaranduba foi a que apresentou valor abaixo da média, explica-se pelo fato de que esta é uma espécie que ao serrar produz menos peças, o que resulta em baixo rendimento. 
FREITAS, N. de N.B.; SANTOS, I.B.; PEREIRA, P.C.G. Rendimento de madeiras nativas.

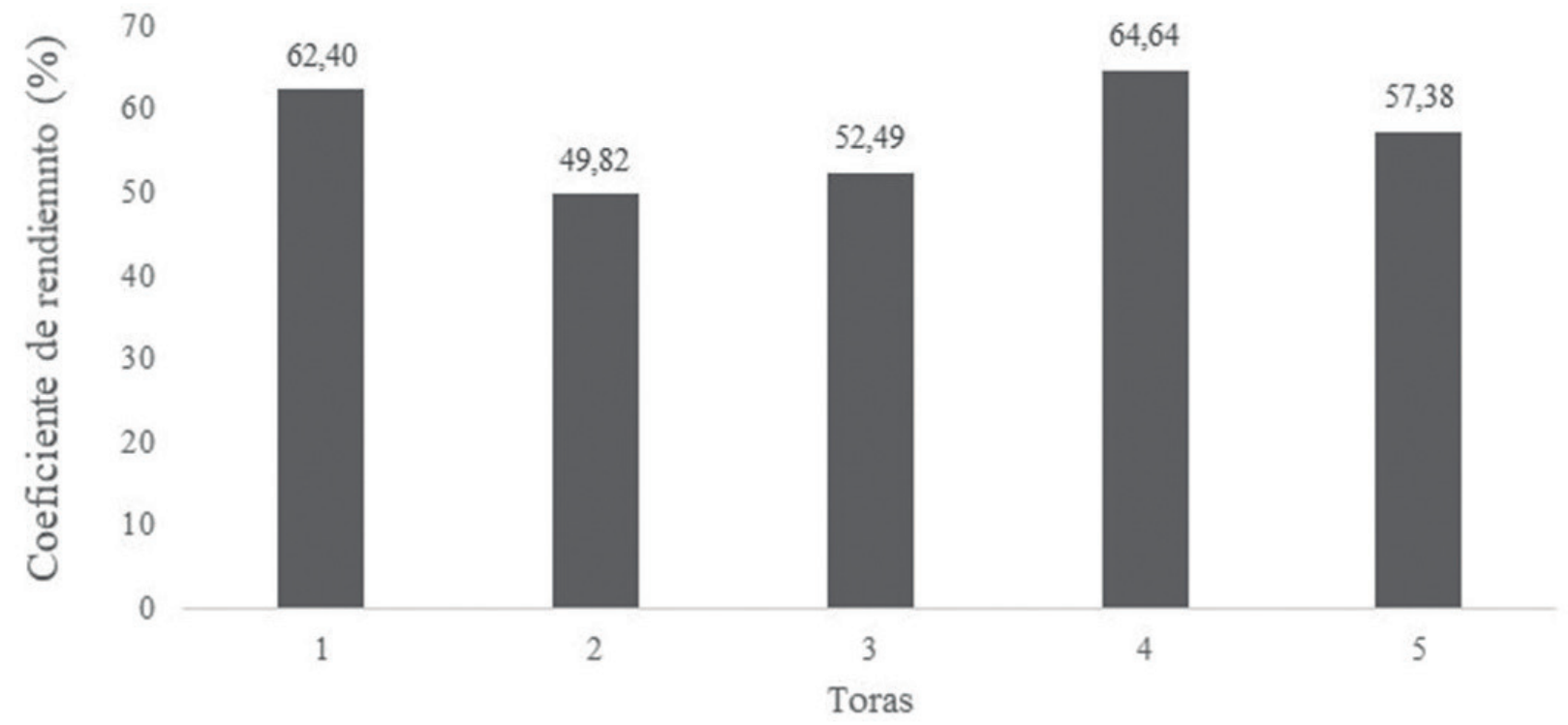

Figura 3. CRV da cupiúba por tora.

Figure 3. Coefficient of volumetric yield of cupiúba per log.

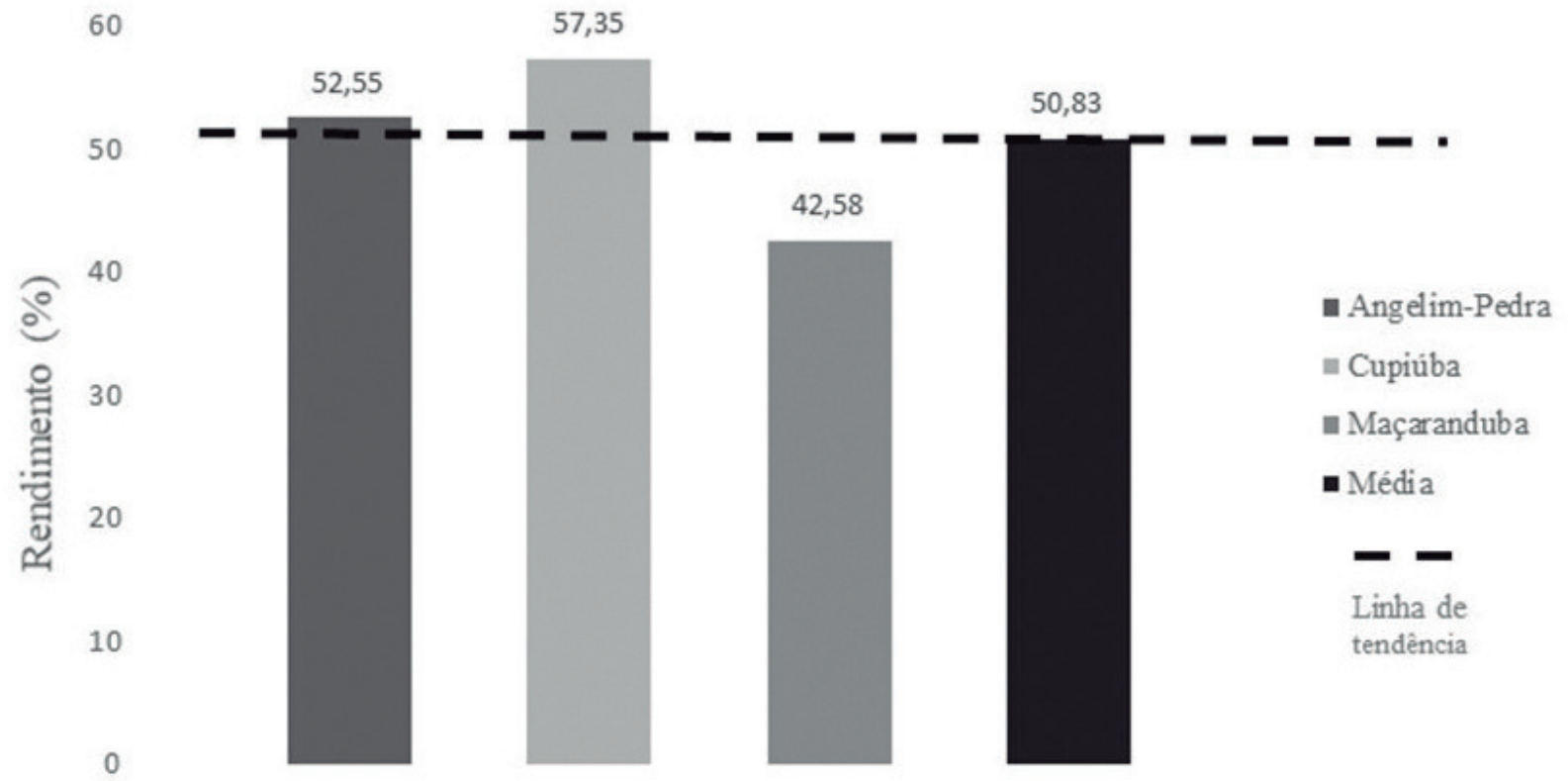

Figura 4. Rendimento médio em madeira serrada e rendimento volumétrico do angelim-pedra, cupiúba e maçaranduba.

Figure 4. Average yield in sawed wood and volumetric yield of angelim-pedra, cupiúba and maçaranduba. 
FREITAS, N. de N.B.; SANTOS, I.B.; PEREIRA, P.C.G. Rendimento de madeiras nativas.

O valor de rendimento $42,58 \%$ pode estar relacionado ao processo de desdobro, principalmente na realização da destopa da madeira serrada (esquadrejamento), bem como pode estar associado às características das espécies como presença de oco (Gerwing et al., 2000).

Ao analisar o rendimento do processamento de toras em dez serrarias e duas laminadoras, em Paragominas/PA, Gerwing et al. (2000) encontraram rendimento médio total de $39 \%$ para as laminadoras, $36 \%$ de rendimento de serrarias, que serram para o mercado local, e $32 \%$ para as empresas que produzem para a exportação. Os autores explicam que o resultado dos rendimentos foi influenciado por fatores como variação na espessura da madeira processada, resultante da utilização de equipamentos de processamento inadequados ou gastos, causando perdas de $\geq 8 \%$ do volume durante o processo de desdobro.

Comparando-os, o rendimento da madeireira em estudo apresentou-se satisfatório visto que as toras usadas para as amostras se encontravam com características favoráveis ao processamento. Somente as toras 2 e 4 da espécie maçaranduba apresentaram ocos, contudo, seus ocos foram descontados no cálculo para obtenção do volume, o que não interferiu no resultado do rendimento volumétrico. Salienta-se que os equipamentos obsoletos utilizados e a falta de mão de obra qualificada, podem ter influenciado no valor do rendimento da empresa, significando não ser o valor real do rendimento da madeireira em estudo, porém, para confirmar e concretizar esta afirmativa é necessária a realização de outro estudo em que se viabilize a eficácia da capacidade dos equipamentos utilizados no desdobro de toras nas empresas.

As médias dos rendimentos não apresentaram diferença significativa no método de cubagem verificado entre as espécies, mas diferem significativamente entre os métodos de cubagem, isto é, comparando um método com o outro (Tabela 3). Isso pode ser explicado, pois ao se avaliar o volume da tora pelo método de Francon, esquadreja-se a madeira retirando-se as costaneiras, consequentemente, apresenta diferença no volume e rendimento ao comparar com o método de cubagem Smalian, o qual obtém os dados através das medições das circunferências, não desprezando as costaneiras.

Angelim-pedra apresentou maior rendimento médio Francon (67,08\%) e cupiúba possuiu maior rendimento médio Smalian (57,35\%). Cunha et al. (2015) realizaram também análise estatística em rendimento médio geométrico de duas espécies de eucalipto em diferentes classes diamétricas através do teste de Tukey, os autores encontraram alta média de rendimento, em que o Eucalyptus grandis apresentou maior rendimento médio $(56,28 \%)$ em relação a Eucalyptus benthamii (52,96\%).

Tabela 3. Médias de rendimento volumétrico por métodos diferentes de cubagens.

Table 3. Averages of volumetric yield in different cubing methods.

\begin{tabular}{ccc}
\hline \multicolumn{3}{c}{ COEFICIENTE DE RENDIMENTO VOLUMÉTRICO (\%) } \\
\hline ESPÉCIES & SMALIAN & FRANCON \\
\hline ANGELIM -PEDRA & $52,55 \mathrm{a}$ & $67,08 \mathrm{~b}$ \\
CUPIÚBA & $57,35 \mathrm{a}$ & $62,43 \mathrm{~b}$ \\
MAÇARANDUBA & $42,59 \mathrm{a}$ & $48,56 \mathrm{~b}$ \\
\hline MÉDIA & 50,83 & 59,36 \\
\hline
\end{tabular}

Médias seguidas pela mesma letra, não diferem estatisticamente entre si, segundo o teste de Tukey (95\% de probabilidade).

Similarities with the letter are not statistically different from each other, according to Tukey test (95\% probability). 
FREITAS, N. de N.B.; SANTOS, I.B.; PEREIRA, P.C.G. Rendimento de madeiras nativas.

Os altos valores de rendimentos encontrados neste trabalho ocorreram pela grande quantidade de peças que cada tora gerou, destas se destacam as ripas, que são produtos comercializados pela madeireira. Baseando-se na resolução CONAMA n 474/2016 (Brasil, 2016), cada peça deve ser inclusa no volume de madeira serrada para determinar o CRV, as ripas, por serem um produto, foram utilizadas no volume de madeira serrada, intervindo no valor do rendimento.

Ao analisar os rendimentos volumétricos por diferentes métodos de cubagem, observou-se que o método de Francon foi aquele que apresentou maior rendimento nas três espécies estudadas como demonstrado na Tabela 2. Ressalta-se que o alto rendimento é gerado pelo aproveitamento das costaneiras, transformando-as em produtos ou subprodutos, também é importante mencionar que o volume de madeira cubada por método de Francon sempre apresentará CRV superior aos demais métodos, pois o volume real da tora é subestimado, por este motivo o rendimento das espécies que utilizam o volume da tora por método de cubagem Francon apresentará o volume do produto verdadeiro e o volume da tora subestimado, pois ao cubar a tora descontam até $30 \%$ das extremidades e, consequentemente, depois as usam, gerando produtos como ripas e caibros.

\section{CONCLUSÕES}

O rendimento bruto do processo de desdobro para as três espécies estudadas por diferentes métodos de cubagem foi superior ao limite previsto pela resolução CONAMA $n^{\circ} 474 / 2016$ que é de $35 \%$, no método de Smalian os valores médios apresentados foram de 50,83\%, enquanto no de Francon foram de 59,36\%.

A espécie cupiúba apresentou valor maior de rendimento, $57,35 \%$, quando utilizado o método de Smalian e a maçaranduba com, $42,58 \%$, o menor percentual.
No método de Francon, o maior coeficiente observado foi para o angelim-pedra, $67,08 \%$, e o menor valor foi apresentado por maçaranduba, 52,64\%.

Deve-se atentar para o método utilizado para a cubagem de toras, de forma que não se obtenham rendimentos que não condizem com a realidade e, consequentemente, divergência de informações quanto ao CRVs.

\section{REFERÊNCIAS BIBLIOGRÁFICAS}

BATISTA, D. et al. Desempenho operacional de uma serraria de pequeno porte do município de Alegre, Espírito Santo, Brasil. Revista Floresta, v. 45, n. 3, p. 487 - 496, 2015.

BRASIL. Ministério do Meio Ambiente - MMA. Conselho Nacional de Meio Ambiente - CONAMA. Resolução $\mathrm{n}^{\circ} 411$ de 06 de maio de 2009. Dispõe sobre o procedimento para inspeção de indústrias consumidoras ou transformadoras de produtos e subprodutos florestais madeireiros de origem nativa, bem como os respectivos padrões de nomenclatura e coeficientes de rendimentos volumétricos, inclusive carvão vegetal e resíduos de serrarias. Disponível em: <http://www.mma.gov.br/ port/conama/legiabre.cfm?codlegi $=604>$. Acesso em: 2 set. 2017.

Resolução $\mathrm{n}^{\circ} \quad 474$ de 06 de abril de 2016. Altera a Resolução n $n^{\circ} 411$ de 06 de maio de 2009, que dispõe sobre o procedimento para inspeção de indústrias consumidoras ou transformadoras de produtos e subprodutos florestais madeireiros de origem nativa, bem como os respectivos padrões de nomenclatura e coeficientes de rendimentos volumétricos, inclusive carvão vegetal e resíduos de serrarias, e dá outras providências. Disponível em: $<\mathrm{http}: / / \mathrm{www} \cdot \mathrm{mma}$. gov.br/port/conama/legiabre.cfm?codlegi $=720>$. Acesso em: 2 set. 2017. 
CUNHA, A.B. da et al. Avaliação do rendimento em madeira serrada de Eucalyptus benthamii e de Eucalyptus grandis por meio do desdobro tangencial e radial. Revista Floresta, v. 45, n. 2, p. 241-250, 2015.

DANIELLI, F. Modelagem do rendimento no desdobro de toras de Manilkara spp. (Sapotaceae) em serraria na Nova Fronteira Madeireira do Estado de Roraima, Brasil. 2013. $82 \mathrm{f}$. Dissertação (Pós-graduação em Ciências de Florestas Tropicais) - Instituto Nacional de Pesquisas da Amazônia - INPA, Manaus.

GARCIA, F. Rendimento operacional de uma serraria com a espécie cambará (Qualea albiflora Warm.) na Região Amazônica. Botucatu: Universidade Estadual Paulista - UNESP, 2013. 69 p.

GERWING, J. et al. O rendimento no processamento de madeira no Estado do Pará. Belém: IMAZON, 2000. 38 p.

HIRAMATSU, N. Equações de volume comercial para espécies nativas na região do Vale do Jari, Amazônia Oriental. 2008. Dissertação (Mestrado em Ciência Florestal) - Universidade Federal do Paraná - UFPR, Curitiba.

INSTITUTO DO HOMEM E MEIO AMBIENTE DA AMAZÔNIA - IMAZON. A atividade madeireira na Amazônia brasileira: produção, receita e mercados. Belém: Serviço Florestal Brasileiro, 2010. 20 p.

IWAKIRI, S. Rendimento e condições de desdobro de 20 espécies de madeiras da Amazônia. Manaus, Amazônia, Acta Amazônica, v. 20, n. único, p. 271-281, 1990.

JUVENAL, T.; MATTOS, R. O setor florestal no brasil e a importância do reflorestamento. BNDS Setorial, n. 16, p. 3-29, 2002.
MANHIÇA, A. Rendimento e eficiência no desdobro de Pinus sp. utilizando modelos de corte numa serraria de pequeno porte. 2010. 466 f. Dissertação (Pós-Graduação em Engenharia Florestal) - Setor de Ciências Agrárias, Universidade Federal do Paraná, Curitiba.

MELO, L.E.L. et al. Resíduos de serraria no Estado do Pará: caracterização, quantificação e utilização adequada. Floresta e Ambiente, v. 19, n. 1, p. 113-116, 2012.

MURARA JUNIOR, M.; ROCHA, M.P.; TIMOFEICZYK JUNIOR, R. Rendimento em madeira serrada de Pinus taeda para duas metodologias de desdobro. Revista Floresta, v. 35, n. 3, p. 473-483, 2005.

MURARA JUNIOR, M.; ROCHA, M.P.; TRUGILHO, P.F. Estimativa do rendimento em madeira serrada de Pinus para duas metodologias de desdobro. Floresta e Ambiente, v. 20, n. 4, p. 556-563, 2013.

PARÁ. Secretaria de Estado de Meio Ambiente e Sustentabilidade - SEMAS. Comercialização de madeira por tipo de produto: período de $-1 /-1 / 2015$ até 31/12/2015. Belém, 2016. 5 p.

PIMENTEL GOMES, F. Curso de estatística experimental. 14. ed. Piracicaba: Desgaspari, 2000. $447 \mathrm{p}$.

SANTOS, E.K.H. et al. O rendimento de duas espécies florestais amazônicas após desdobro primário. In: CONGRESSO BRASILEIRO DE CIÊNCIA E TECNOLOGIA DA MADEIRA, 3., 2017, Florianópolis. Resumos... Campinas: Galoá, 2018. Disponível em: <https://proceedings. science/cbctem/trabalhos/o-rendimento-de-duas -especies-florestais-amazonicas-apos-desdobro -primario>. Acesso em: 21 ago. 2016. 
FREITAS, N. de N.B.; SANTOS, I.B.; PEREIRA, P.C.G. Rendimento de madeiras nativas.

TONINI, H.; FERREIRA, L.M. Rendimento em madeira serrada de cupiúba (Goupia glabra), caferana (Erisma uncinatum) e angelim-pedra (Dinizia excelsa). Boa Vista: EMBRAPA Roraima, 2004. Não paginado. (Comunicado Técnico, 07).

VITAL, B.R. Planejamento e operação de serrarias.

Viçosa, MG: UFV, 2013. 211 p. 